George N. Frantziskonis*

\title{
Temporal scaling in fatigue life of materials and incorporation of temporal events in Paris's law
}

\begin{abstract}
Temporal scaling in mechanical strength of materials is vital for long-term effects such as fatigue. The fatigue crack length $\alpha$ is related to the change in the stress intensity factor $\Delta K$ by the omnipresent Paris's law, which works well for cyclic fatigue of specific frequency and amplitude. The paper considers time scaling in fatigue and through it incorporates the effects of temporal events such as unexpected or accidental loads, impact loads, and rare events such as earthquake loads. This is achieved by theoretically incorporating the effects of delta-function type loads into fatigue. Since the time-scale decomposition of such a load contains information at all scales, the theoretical framework is easily extended to include general types of loads.
\end{abstract}

Keywords: fatigue; Paris’s law; temporal scaling.

*Corresponding author: George N. Frantziskonis, Civil Engineering and Engineering Mechanics, Materials Science and Engineering, University of Arizona, Tucson, AZ 85721, USA,

e-mail: frantzis@email.arizona.edu

\section{Introduction}

Most science and engineering problems involve a breadth of temporal and spatial scales. Information about a problem may be collected via experiments or observations or may be generated via models. The relevant data are or can be vast, to the point that their management and interpretation become problematic. This would be the case for fatigue loading for example, if it were feasible or practical to be studied experimentally or computationally for the entire duration of the fatigue life of a structural component. It is the scaling of such problems that allows us to study them and model them either fundamentally or empirically in a way that the relevant data are relatively small. Presumably, the scaling allows a model or experiment at particular spatial and temporal scales to be able to provide information at all scales of interest. However, this presumption-based framework is not well established and this leads to empirical or semi-empirical models such as Paris's law for fatigue of materials.
In an accompanying paper [1], spatial scaling in polycrystalline materials is considered and how it relates to size and Hall-Petch effects. Even though spatial scaling and temporal calling are strongly interconnected, for specific time scales temporal scaling can be ignored as was done in Ref. [1]. This is not the case for problems such as fatigue where temporal scaling is important since fatigue events occur at time scales ranging from subsecond to years.

\section{Spatial scaling}

Spatial scaling in mechanical strength of materials addresses the relation between materials structure at various scales and its effects on measures such as strength at various scales. There is a strong interplay between structure and properties where typically meso/microscales are addressed in materials science and solid-state physics, whereas large scales and continuum behavior are addressed in design engineering. It is very difficult and perhaps impractical to address all scales, from atomic to continuum, and the structural changes that occur at each scale during the service life of a material or structural component. For polycrystalline materials, an important range of scales is from that of the average grain size to the continuum one and that range of scales was addressed in Ref. [1].

\section{Temporal scaling and the coupling of space and time}

Temporal scaling in mechanical strength of materials is important for long-term effects, primarily fatigue yet also for creep and relaxation. For fatigue, which is clearly a stochastic process showing considerable scatter even in controlled environments, the fatigue crack length $a$, and thus its statistics, is related to the change in the stress intensity factor $\Delta K$ by the omnipresent Paris's law [2, 3]. Information on the crack(s) size and configuration may be present at a multiplicity of scales, and recent work [4] sets a basis 
to study the scaling of fatigue and whether it scales in a way similar to the spatial scaling addressed in Ref. [1].

In particular, there are two distinct time scales in fatigue that interact with each other. At the short time scale, i.e., that of a few load cycles, during each cycle small structural changes occur in the material, typically modeled through a crack increase d $a$. Significant structural changes (eventually leading to failure) occur at a large number of cycles or at time scales orders of magnitude larger than the period of a single cycle. Fusion of information on structural changes at a small number of cycles and at large time scales provides a unified description of the fatigue problem. Yet, at small time scales (e.g., over a few cycles), structural changes occur at small (atomic to micrometer) spatial scales and, at large time scales, structural changes occur at the continuum level (millimeter and above for polycrystalline metals). Figure 1 shows a schematic of the time scales and corresponding structural changes during fatigue, where structural changes are quantified through the crack length $a$.

It is interesting that attempts to link the time scales in a multiscale fashion have appeared in the recent literature $[5,6]$. In these studies, the objective was to obtain the coarse time scale state evolution equation using the wavelet representation of the strain field at the scale of interest, i.e., at cycle $N$. The wavelet decomposition of the strain field contains information on smallscale (cycle) response through the wavelet basis functions and transforms the large-scale material response into a cycle scale problem, which is monotonically increasing. This is a sort of fusion/fission process of data [7] (the strain field here and its relation to fatigue damage), yet a single scale model is used for both the small- and large-scale evolution of damage, in particular crystal plasticity.

The structural changes or crack propagation occurring at small time scales is mostly governed by atomistic events, whereas that at large time scales is governed by continuum plasticity. Thus there is a coupling of space and time [8], and this can be studied along the lines of Frantziskonis [1] with time being an additional dimension in the problem. However, in this work, time scaling is "isolated", i.e., examined by itself, keeping in mind the coupling of space and time.

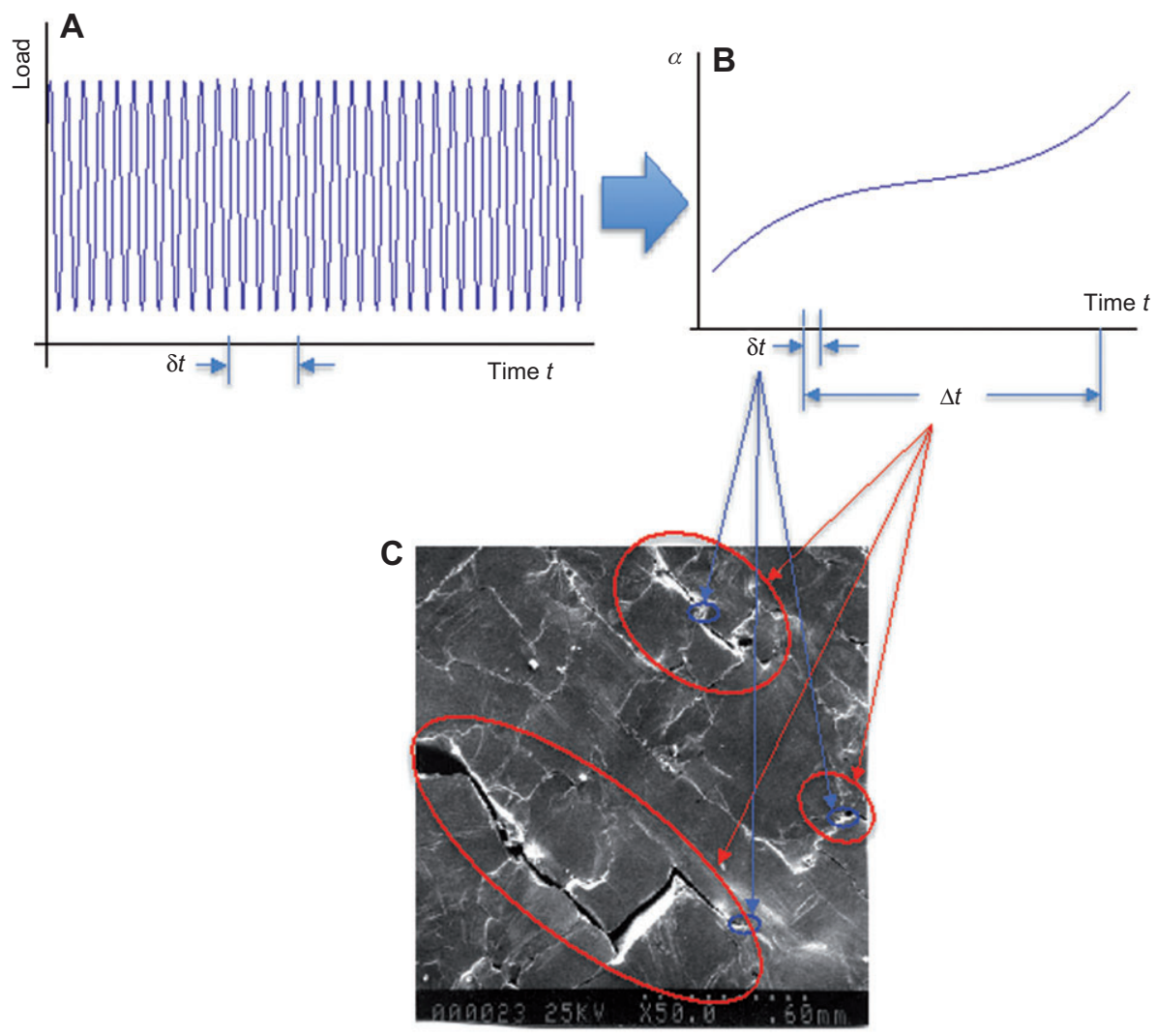

Figure 1 Time scales and example of structural changes during fatigue loading of a polycrystalline material. (A) Schematic of the load vs. time during fatigue. (B) Schematic of corresponding changes in material state variables realized through $a$ and short time scale $\delta t$ and large time scale $\Delta t$. (C) Typical electron microscopy image of a fatigue-damaged polycrystalline steel, and example changes in state variables over $\delta t$ (inside the blue ellipses) and $\Delta t$ (inside the red ellipses). 


\section{Effects of structural changes in polycrystalline metals - spatial scaling and structural changes}

Structural changes in polycrystalline metals during their service life are typically embedded in the length of a fatigue crack, $a$, and incorporate its effect on the relevant stress intensity factor. A small increase in crack length, $\delta \alpha$, can be considered as the introduction of a delta function on the deformation field. This, in fact, is a structural change at the finest scale possible, and its effect, as shown in Figure 2, is present at all scales. Thus, by considering the change in crack length, $\delta \alpha$, classical fatigue analysis is indirectly performing a multiscale analysis process. This is an elegant way to "homogenize" structural changes such as defects at the atomic scale into a single parameter, $\delta \alpha$, which has implications at all scales.

Classical fatigue analysis does not consider the link of spatial to temporal scaling, and successful research in this area, as attempted in this work, will furnish a major leap forward in the area of fatigue life. In other words, for cycling fatigue of specific frequency and amplitude, the classical fatigue theory works well and is naturally multiscale. Next, a way to incorporate temporal scaling in fatigue is described.

\section{Temporal scaling and structural changes}

Even though fatigue models as described above work relatively well for consistently cycling load cases, temporal events deviating from the cycling load affect the behavior of materials. Examples of such events include accidental or rare loads such as ones from earthquakes for civil structures, impact loads from accidents, etc. Temporal loads introduce a temporal scaling in the problem, which is directly connected to spatial scaling. For example, a delta-function type load contains information and load components at all temporal scales; thus it has implications on the relevant spatial scales. Figure 3B shows the wavelet scalogram of the delta-function shown in Figure 3A. As can be seen, the delta function introduces temporal
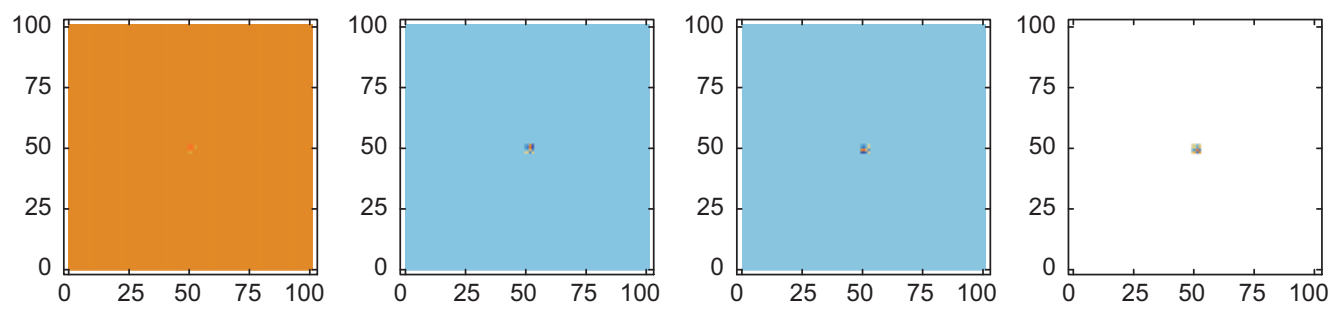

Figure 2 Illustration of the impact of a delta function structural change, such as the (small) increase in the length of a fatigue crack, on a constant field. In a $100 \times 100$ constant field, a delta function was introduced at the center. The figure shows the density plot of the wavelet transform of the field at four scales from coarse (left) to fine (right).
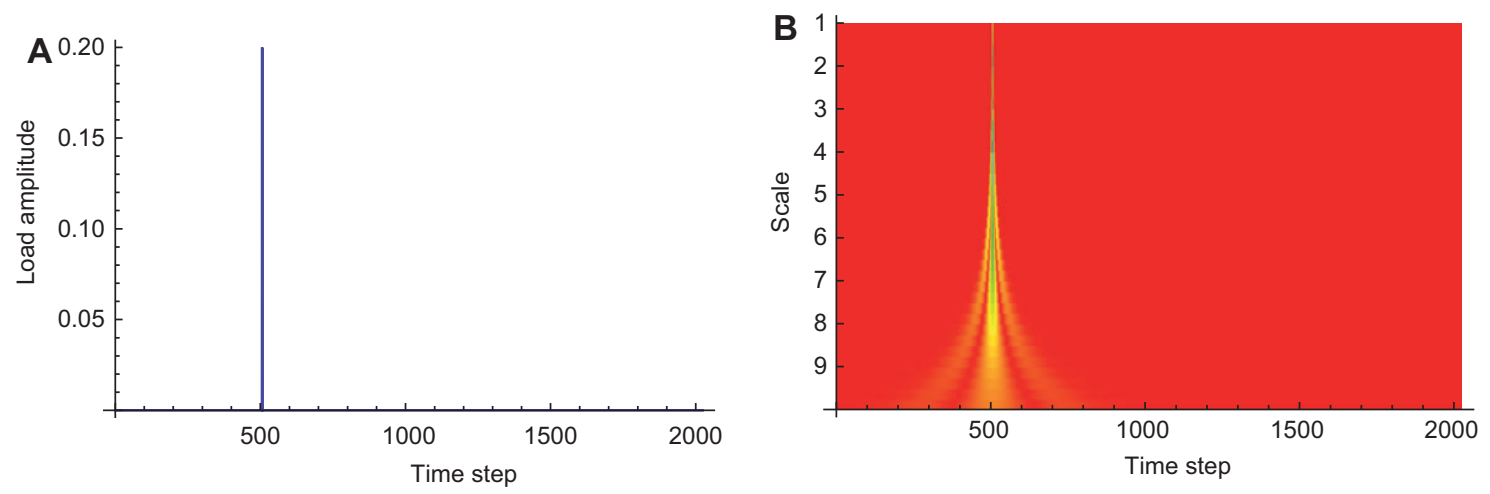

Figure 3 (A) A delta-type function shown in a time vs. mechanical load amplitude plot. (B) The wavelet scalogram of the function in (A) showing, in yellow, the existence of wavelet coefficients of significant energy in time vs. scale. Scale 1 is the smallest one equal to one time step, and scale 10 is the largest scale of the order of the entire time interval. 
disturbances at all scales. It appears, from a first glance, however, that at larger scales the effects of a delta function appear in time before the actual delta-type event occurs. This, of course, does not mean that it affects events in the past, but it is a result of transforming the time-dependent problem in the wavelet domain.

Using a delta-type function for the representation of temporal loads from rare or non-regular events has many advantages. First, the precise or even the rough estimate value and nature of such loads are rarely known; a deltatype function can at least provide a conservative estimate for the maximum temporal load. Second, a deltatype function provides a conservative approach to the study of the effects of rare or unexpected loads since it contains information at all scales. Third, every function can be decomposed into a sequence of delta-type functions. Thus, in the proposed study, temporal scaling will be examined mostly by studying the effects of delta-type functions for the loads on a structure resulting from rare or unexpected events.

We start with the classical Paris's law

$$
\frac{d \alpha}{d N}=c(\Delta \sigma \sqrt{\pi \alpha})^{m}
$$

where $N$ denotes the number of cycles, $\Delta \sigma$ is the stress amplitude of a cyclic load, and $c$ is a constant. The solution to this equation reads

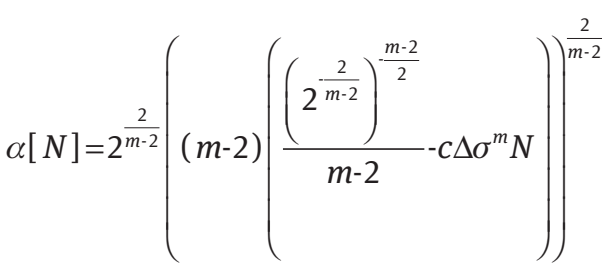

We consider a continuous wavelet to be of the form ${ }^{1}$

$$
\psi(t)=\left(t^{2}-1\right) \exp \left(-\frac{1}{2} t^{2}\right)
$$

and a cycling load function of the form

$$
\sigma(t)=\frac{1}{2} A \sin (\omega t)
$$

where $A$ denotes amplitude and $\omega$ denotes frequency, such that $\Delta \sigma=A$. The wavelet transform of the cycling load

1 A study on the influence of the wavelet used resulted in that the process is rather insensitive to it, i.e., the main results and trends remain unaltered by the wavelet choice. This holds true for many continuous wavelets including ones based on derivatives of Eq. (3). function in Eq. (4) using Eq. (3) and the definition of the wavelet transform,

$$
T_{\sigma}(b, a)=\frac{1}{\sqrt{a}} \int_{-\infty}^{\infty} \sigma(t) \psi\left(\frac{t-b}{a}\right) d t,
$$

yield

$$
T_{\sigma}(b, a)=\frac{1}{2} \sqrt{2 \pi} A a^{2} \omega \cos (\omega b) \exp \left(-\frac{1}{2} \omega^{2} a^{2}\right),
$$

with $a$ denoting scale and $b$ denoting the translation in time variable

When a delta function is superimposed on the cycling load, the small scales $a$ are the ones with the high energy in the vicinity of the delta function. Given that, and expanding the $a^{2} \exp \left(-\frac{1}{2} \omega^{2} a^{2}\right)$ term in Eq. (6) into a Taylor series around $a=0$ and ignoring higher order terms, it follows that

$$
T_{\sigma}(b, a) \sim A a^{2} \omega \cos (\omega b)
$$

Performing the inverse wavelet transform based on Eq. (7), the term $a^{2}$ is eliminated (the inverse wavelet transform involves a term $\sim 1 / a^{2}$ ), and this suggests the following form for the temporal evolution of the crack length $\alpha$ in the vicinity of a delta-function type disturbance in the $\operatorname{load} \sigma$

$$
\frac{d \alpha}{d N}=c_{1}\left(\Delta \sigma_{1} \sqrt{\pi \alpha}\right)^{m} .
$$

where $\Delta \sigma_{1}$ includes the disturbance due to the delta type function. This, i.e., Eq. (8), cannot be solved analytically for $\Delta \sigma_{1}$ containing a delta function or step function (wide delta function) term, but its numerical integration is straightforward. Of course, its rationale needs to be verified by experimental information.

We consider the following values for the constants, inspired by typical values used in metal fatigue, $m=2.8$, $c_{1}=10^{-10}, \Delta \sigma=1000, \Delta \sigma_{1}=\Delta \sigma+1000$, and the initial value of the fatigue crack length is considered to be unity, which implies a fast increase of the fatigue crack length with the number of cycles. With these values, a jump in the stress over that of the cycling stress of 1000 units of stress is considered. The jump in the considered example starts at the 20th fatigue cycle, thus at $N=20$, and lasts for 10 time steps [the 10 time steps avoid numerical instability in solving Eq. (8) numerically which occurs for a small period such as 1 or 2 time steps]. Figure 4 shows the length of the crack as a function of load cycles $N$ for the cases of cycling loading and of cycling loading and an unexpected load as described above. For the cycling load, the crack 


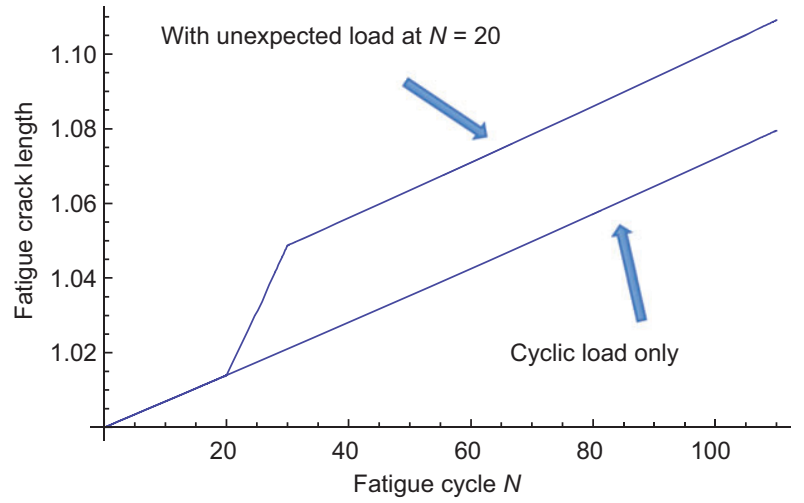

Figure 4 Fatigue crack length as a function of number of fatigue cycles for two stress cases, one with only cycling load and one with cycling load plus an unexpected load commencing at load cycle number 20 and lasting for 10 cycles.

length advances, relatively fast for the constants and stresses considered here for illustration and for the large initial crack length.

This concurs with experimental results that indicate fatigue cracks continue to grow at the expected rate after a change in load is imposed $[9,10]$. The jump in the fatigue crack length is that from the imposed load, considering it here, without loss of generality, to be of the same type as the cycling load, and being applied in a quasi static fashion, i.e., ignoring any type of dynamic load effects. Furthermore, the crack propagation stability is assumed to be the same as that for the cycling load. These assumptions can be relaxed for a general problem studied numerically under the present framework.

In engineering practice, the decision whether to rehabilitate or replace a structural component or an entire structure is part of daily life. Such decisions are typically based on visual or non-destructive inspection of the materials. Sometimes, a study will be conducted to assess the performance of a structure or structural component containing defects identified from the inspections. For fatigue life, the remaining life may be estimated by mapping the identified defects to a crack length and then performing fatigue analysis assuming a certain cycling load during the remaining life.

The present and current material inspection methods identify defects at large (continuum) scales. Thus, in a sense, the process of assessing the remaining life of a structure or structural component is based on large spatial and corresponding large temporal scales. This approach may be sufficient provided the structure is not subjected to rare loads, especially ones of extreme nature. High time rate loads (such as delta- or step-function type ones) affect all scales. Thus, the methodology presented herein has potentially important consequences in studying the remaining life of a structure or structural component robustly, and from that, more informed decisions on whether to rehabilitate or replace a structure can be made.

In concluding the present report, a robust process for the prediction of fatigue behavior of materials under cycling load and temporal spikes in load due to random events is presented. As shown in Figure 5, a delta-function type load in addition to a cycling load has implications at all temporal scales and, correspondingly, at all or a wide range of spatial scales. As shown in Figure 5B, the cycling load contains information at a fixed range of scales, i.e., in the vicinity of the period of the cycling load. However, a delta-function spike spreads information at all scales, above and below the ones in the vicinity of the cycling period. (Note that the intensity near the boundaries in Figure $5 \mathrm{~B}$ is due to boundary effects in the scalogram.) Evaluation of the work presented herein for such load provides the basis for studying any type of fatigue load including stochastic ones for which there is a rich literature on experimental data [11, 12].
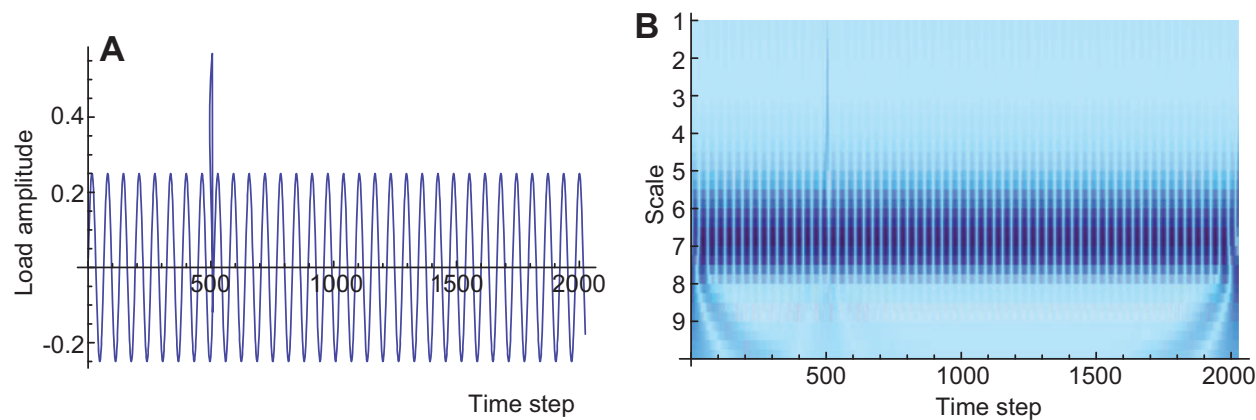

Figure 5 (A) A delta-type function superimposed on a cycling one shown in a time vs. mechanical load amplitude plot. (B) The wavelet scalogram of the function in (A) showing, in dark blue, the existence of wavelet coefficients of significant energy in time vs. scale. Scale 1 is the smallest one equal to one time step, and scale 10 is the largest scale of the order of the entire time interval. 


\section{References}

[1] Frantziskonis G. J. Mech. Behav. Mater. 2013, 21, 175-180.

[2] Paris PC, Gomez MP, Anderson WE. Trend Eng. 1961, 13, 9-14.

[3] Suresh S. Fatigue of Materials. Cambridge University Press: Cambridge, 1998.

[4] Frantziskonis G, Matikas TE. Mater. Trans. 2009, 50, 1758-1767.

[5] Anahid M, Chakraborty P, Joseph D, Ghosh S. Model. Simul. Mater. Sci. Eng. 2009, 17, 064009.

[6] Joseph DS, Chakraborty P, Ghosh, S. Comp. Methods Appl. Mech. Eng. 2010, 199, 2177-2194.

[7] Frantziskonis G. Data fusion and fission for information at particular scales and for reducing data set size-application to probabilistic scaling in material strength, University of Arizona,
Civil Engineering and Engineering Mechanics Report No. CEEM-2013.4, September 2012.

[8] Sih G, ed. Multiscaling in Molecular and Continuum Mechanics: Interaction of Time and Size from Macro to Nano: Application to Biology, Physics, Material Science, Mechanics, Structural and Processing Engineering. Springer-Verlag, 2007 (Google eBook).

[9] Taneda M, Koibuchi K, Matsukawa Y. Bull. Jpn. Soc. Mech. Eng. 1971, 14, 534-540.

[10] Frost NE, Marsh KJ, Pook LP. Metal Fatigue. Dover: Oxford, 1999.

[11] Elements of Metallurgy and Engineering Alloys, Chapter 14, Fatigue. ASM International, 2008.

[12] Nieslony A. J. Theor. Appl. Mech. 2010, 48, 233-254. 